\title{
A population-based study of cystic white matter injury on ultrasound in very preterm infants born over two decades in Nova Scotia, Canada
}

\author{
Satvinder Ghotra ${ }^{1} \cdot$ Michael Vincer ${ }^{1} \cdot$ Victoria M. Allen $^{2} \cdot$ Naeem Khan $^{3}$
}

Received: 13 May 2018 / Revised: 27 November 2018 / Accepted: 27 November 2018 / Published online: 14 December 2018

(c) Springer Nature America, Inc. 2018

\begin{abstract}
Objective To identify the temporal trends, risk factors and outcomes of cystic white matter injury (WMI) detected by ultrasound in a population-based cohort of very preterm infants (VPI) with a minimal risk of selection bias.

Study design All live-born VPIs between 22 and <31 weeks gestational age born in Nova Scotia, Canada from 1993 to 2013.

Results Cystic WMI was identified in $87(7 \%)$ out of 1184 eligible infants. The gestational age and mortality adjusted prevalence of cystic WMI decreased over time $(p=0.04)$. In multivariable analysis, chorioamnionitis, antenatal steroids, admission hypothermia, ventilator support, inotropes, and non-Coagulase-negative Staphylococcal and fungal infections were independently associated with cystic WMI. Cerebral palsy was the most common disability in the survivors, however, half of the survivors had none or mild disability.

Conclusions This cohort study demonstrated a decreasing trend in the incidence of cystic WMI and reported populationbased neurological outcomes with cystic WMI, which is important for health-care planning and parental counseling.
\end{abstract}

\section{Introduction}

Advances in maternal and newborn care have significantly improved the survival in preterm infants; however, chronic neurological disabilities continue to be a major health issue in survivors [1]. Of $\sim 60,000$ very low birthweight $(<1500 \mathrm{~g})$ infants born every year in the United States, 5-10\% develop substantial motor defects and 25-50\% develop significant cognitive, behavioral, and learning deficits [1]. According to the Canadian Neonatal Follow-Up Network, a

Supplementary information The online version of this article (https:// doi.org/10.1038/s41372-018-0294-5) contains supplementary material, which is available to authorized users.

Satvinder Ghotra

Satvinder.ghotra@iwk.nshealth.ca

1 Department of Neonatal-Perinatal Medicine, Dalhousie University and the IWK Health Centre, Halifax, NS, Canada

2 Department of Obstetrics and Gynaecology, Dalhousie University and the IWK Health Centre, Halifax, NS, Canada

3 Department of Radiology, Dalhousie University and the IWK Health Centre, Halifax, NS, Canada total of 3700 babies at $<29$ weeks of gestation were admitted to various Canadian Neonatal Intensive Care Units in between 2009 to 2011. Of these, 84\% survived to discharge and of the survivors, $46 \%$ were diagnosed with some degree of neurodevelopmental impairment and $17 \%$ with significant neurodevelopmental impairment at 18 months of corrected age [2]. Cerebral white matter injury (WMI) is an important determinant of these adverse neurodevelopmental outcomes in preterm survivors [3].

Preterm infants are especially vulnerable to WMI owing to a combination of brain immaturity and stressors encountered in the perinatal-neonatal period [4]. WMI in a premature brain can be characterized radiographically as cystic or non-cystic. Cystic WMI, which includes cystic periventricular leukomalacia (cPVL) and, less commonly, porencephaly, is the most severe form of WMI. Cystic PVL represents hypoxic ischemic injury of the preterm brain and pathologically, it is described as the focal necrosis and gliosis of the periventricular white matter with development of cysts in the periventricular area. The distribution of injury is often bilateral and involves the peritrigonal area and anterior and lateral to the frontal horns, referred to as the watershed areas of the preterm brain. Porencephaly is defined as a local area of cyst formation in the brain 
following focal encephalomalacia owing to a localized cerebral insult. It is usually unilateral and can be the late sequelae of a destructive process such as intraparenchymal hemorrhage, infection, or ischemia, where the damaged area of cerebral parenchyma is replaced with cerebrospinal fluid.

Both CPVL and porencephaly are very likely to be associated with neurodevelopmental abnormalities. PVL is known to be highly associated with cerebral palsy (CP) in preterm infants $[4,5]$ and $60-100 \%$ of children with cPVL develop CP [6]. Currently, there is no definitive therapy available for cystic WMI emphasizing the importance of timely identification of associated risk factors and their prevention. Relevant studies on incidence, risk factors, and outcomes of cystic WMI have primarily used retrospective case-control designs, and hence, the possibility of a control selection bias is very high [7-12]. The cohort studies are scarce on this topic and often limited by sample size $[12,13]$. Again, these studies have an inherent selection bias owing to being hospital-based rather than populationbased. This study aimed to address the temporal trends, risk factors, and neurodevelopmental outcomes of cPVL and porencephaly in a large population-based cohort of very preterm infants (VPI) using a prospective database with minimal selection bias.

\section{Methods}

All VPI ( $<31$ weeks' gestational age at birth) born to residents in the province of Nova Scotia, Canada are prospectively enrolled in a population-based provincial database (Perinatal Follow-Up Program (PFUP) database) since 1993. This retrospective cohort study included all live-born VPI, from 22 to $<31$ weeks' gestational age, registered in the PFUP database from 1 January 1993 to 31 December 2013. Infants with severe congenital malformations, chromosomal anomalies, or who died in the first 6 weeks after birth were excluded. The VPI born to mothers residing in Nova Scotia were cared for in the NICU in one of three tertiary care-level hospitals: the IWK Health Centre (Halifax, Nova Scotia); the Cape Breton Regional Hospital (Sydney, Nova Scotia); and the Moncton City Hospital (Moncton, New Brunswick). Some infants were born in one of six regional hospitals and transferred to one of the three above hospitals for NICU care. A population-based design of the database was ensured through following strategies: (1) All three tertiary care-level hospitals and the six regional hospitals were routinely surveyed to identify all VPIs births of Nova Scotia residents; (2) Information was extracted from a detailed chart review of both the maternal and newborn patient records at the IWK Health Centre by the program's database manager, and routinely audited by the Medical Director (MJV) to ensure data accuracy; and (3)
Regular visits for the same type of chart review were performed in the two other tertiary care hospitals and the six regional hospitals by the Medical Director (MJV).

Information on maternal, fetal and neonatal illnesses, and treatments was prospectively entered in the PFUP database. After discharge from the NICU, infants were evaluated in the PFUP on a regular basis to evaluate their neurodevelopmental outcomes by a multidisciplinary team comprising of neonatologists and other allied health professionals, who had full access to patient's previous NICU course. The assessments included between two and six detailed physical and neurological examinations in the first 3 years after birth to determine the presence or absence of $\mathrm{CP}$, a developmental examination including the Bayley Scales of Infant and Toddler Development (II/III version) at 3 years of age, an ophthalmologic examination to evaluate vision and an auditory assessment to evaluate hearing. Data acquired through these evaluations allowed for determination of neurodevelopmental impairment and was also prospectively entered in the PFUP database. CP was defined as a disorder of control of movement or posture secondary to a nonprogressive brain lesion [14]. The five-level Gross Motor Functional Classification System (GMFCS) was used to assess the functional level of CP [15]. Beginning in 2006, Bayley II was replaced with Bayley III for developmental assessment. The cognitive and language composite scores from Bayley III were used to compute the Bayley II mental developmental index (MDI) equivalent scores using the formula: $\mathrm{MDI}=88.8-(61.6 \times[$ language score $/ 100]-1)+$ (0.67×cognitive score) [16]. Disability was defined as presence of $\mathrm{CP}$, MDI score $<85$, or blindness or deafness. Disability was further categorized as (a) mild disability (level 1 GMFCS, and/or, MDI score $<85$ ), (b) moderate disability (level 2 or 3 GMFCS, and/or, MDI score $<70$ ), and (c) severe disability (level 4 or 5 GMFCS, and/or MDI score $<55$, and/or presence of blindness, and/ or, deafness).

After the inclusion criteria were met, information on incidence and potential risk factors of cystic WMI as well as neurodevelopmental outcomes was extracted from the PFUP database. The information on socio-economic status as determined by Hollingshead classification [17] and race was also extracted from the database. The list of risk factors studied and their definitions are provided in Supplementary appendix 1. The diagnosis of cystic WMI was established by standardized serial cranial ultrasounds performed (using $7.5 \mathrm{MHz}$ transducer) within 3 days of birth, at 2 and 6 weeks of age and at term and reported by the local radiologist. The protocol for serial cranial ultrasounds was unchanged for the duration of this investigation except for multiple approaches including the mastoid and anterior fontanel approach introduced in 2010. In this study, cystic WMI was defined as the presence of cPVL or porencephaly. 
The group with cystic WMI was compared to the group with no evidence of cystic WMI on cranial ultrasound. The study was approved by the Research Ethics Board of the IWK Health Centre (Approval \# 1019311).

\section{Statistics}

Categorical and continuous variables were expressed as proportions and means, respectively. Chi-square/Fisher's exact tests were used to compare the categorical variables in groups with and without cystic WMI. Continuous variables across two groups were compared using unpaired $t$ test. Missing data were imputed using hot deck imputation. The risk of cystic WMI was modeled on year, gestational age, and mortality rate using logistic regression. The predicted probabilities of developing cystic WMI from the model for each infant were then averaged by year to yield the annual risk of cystic WMI. A univariate analysis was performed by using a series of single variable logistic regression models to identify the factors associated with occurrence of cystic WMI. Factors with a $p$ value $<0.20$ in the univariate analysis were entered into multivariable risk models for occurrence of WMI. The multivariable models were built separately for each of the three domains (antenatal, perinatal, postnatal) using a backwards selection procedure. Variables with a $p$ value $<0.05$ were retained. The final risk model for cystic WMI was built incrementally by adding blocks of variables retained within each of the three domains in the order of antenatal, perinatal, postnatal. Odds ratio and $95 \%$ confidence interval were reported for each of the risk factors. Statistical analysis was performed using STATA version 12 (StataCorp LP, College station, TX, USA).

\section{Results}

A total of 1501 VPIs $(22-<31$ weeks of gestational age) were born alive to residents of Nova Scotia between 1 January 1993 and 31 December 2013. Figure 1 summarizes the inclusion and exclusion of infants in the study. Of the 140 infants, who died by 6 weeks of age and were excluded from the study, six were identified with cystic WMI based upon an autopsy. Out of 1184 infants found eligible for the study, $87(7.4 \%)$ had WMI. The incidence of WMI decreased over time $(p=0.04)$ after adjusting for gestational age and mortality (Fig. 2).

The racial distribution of eligible participants were as follows: White $(n=571,48.2 \%)$ Black $(n=26,2.2 \%)$, Asian or Pacific Islander $(n=22,1.9 \%)$, Native North American $(n=17,1.4 \%)$, other $(n=5,0.4 \%)$ and unknown $(n=543,45.9 \%)$. Of total, the majority belonged to Hollingshead Class II-IV [Class I (major business and

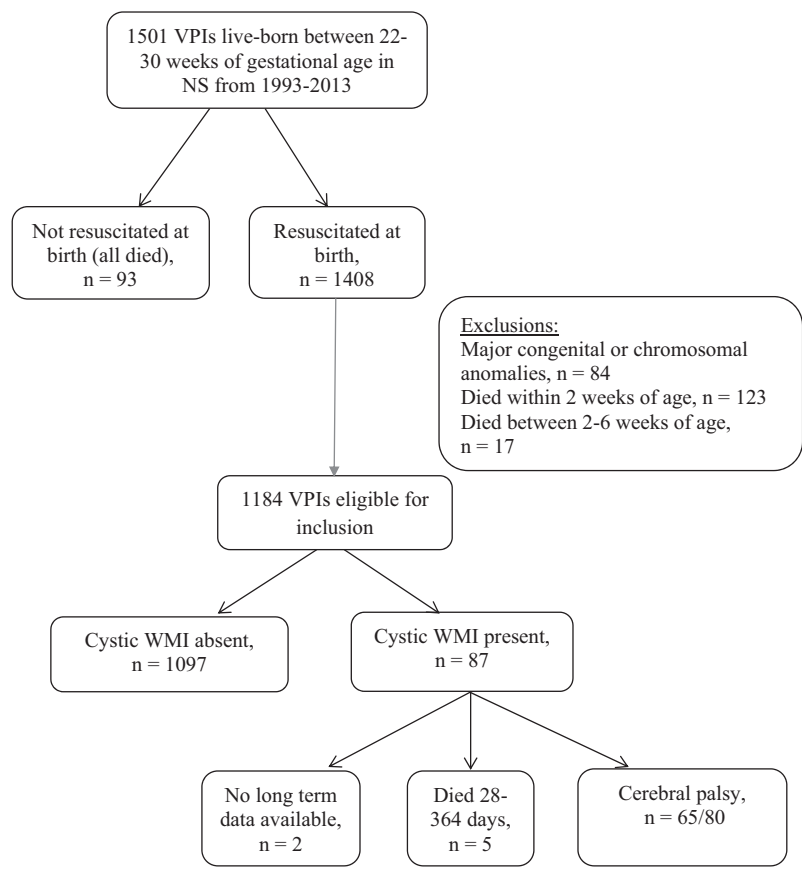

Fig. 1 Flow chart

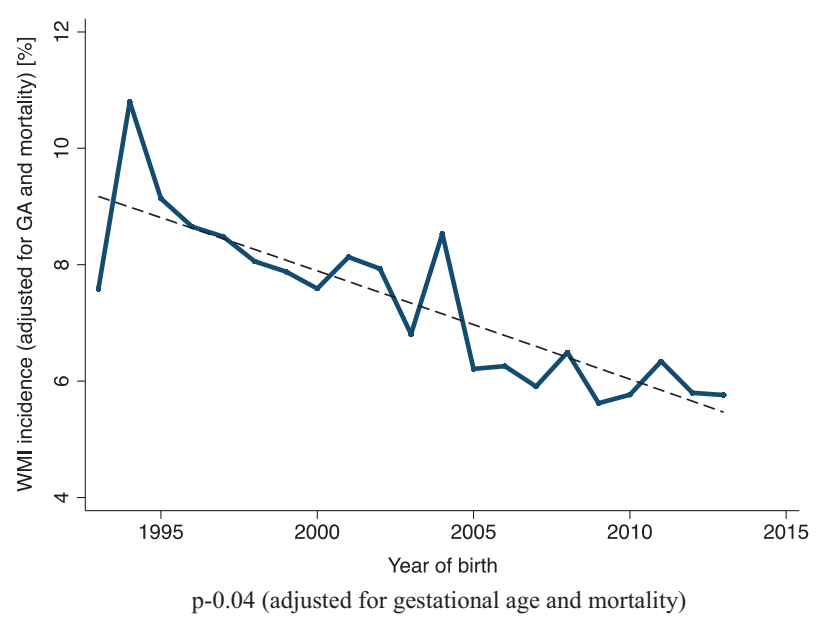

Fig. 2 Adjusted Risk of WMI over time

professional): 92(7.8\%), class II (medium business, minor professional, technical): 322(27.2\%), class III (skilled craftsman, clerical, sales workers): $289(24.4 \%)$, class IV (machine operators, semiskilled workers: 209(17.7\%) and class V (unskilled laborers, menial service workers: 165 (13.9\%) and unknown: 107, 9\%]. Table 1 summarize the antenatal, perinatal, and postnatal characteristics among the WMI and non-WMI groups. Among antenatal factors, histological chorioamnionitis was 2.2 times (95\% CI: 1.2-4.1) more likely in the group with WMI compared with nonWMI group. Women in the WMI group were less likely to have received antenatal steroids for $>48 \mathrm{~h}$ before delivery (OR: 0.2). The rate of multiple births was lower in the WMI category (OR: 0.4 ). The rate of premature and prolonged 
Table 1 Antenatal, perinatal, and ostnatal characteristics of WMI vs non-WMI group

\begin{tabular}{|c|c|c|c|}
\hline Antenatal variables & WMI $\%(n=87)$ & $\begin{array}{l}\text { Non-WMI } \% \\
(n=1097)\end{array}$ & OR $(95 \% \mathrm{CI})$ \\
\hline \multicolumn{4}{|l|}{ Epochs } \\
\hline $2007-2013$ & $31.0(27)$ & $33.6(369)$ & $0.7(0.4-1.3)$ \\
\hline 2000-2006 & $27.6(24)$ & $33.0(362)$ & $0.7(0.4-1.2)$ \\
\hline 1993-1999 & $41.4(36)$ & $33.4(366)$ & Reference \\
\hline Gestational hypertension & $13.8(12)$ & $19.4(213)$ & $0.7(0.4-1.2)$ \\
\hline Histological chorioamnionitis ${ }^{\mathrm{a}}$ & $14.9(13)$ & $7.5(82)$ & $2.2(1.2-4.1)$ \\
\hline Premature rupture of membranes & $25.3(22)$ & $29.3(321)$ & $0.8(0.5-1.4)$ \\
\hline Rupture of membranes $>24 \mathrm{~h}$ & $16.1(14)$ & $22.4(246)$ & $0.7(0.4-1.1)$ \\
\hline Antepartum hemorrhage & $29.9(26)$ & $25.3(277)$ & $1.3(0.8-2.0)$ \\
\hline \multicolumn{4}{|l|}{ Antenatal steroids ${ }^{\mathrm{a}}$} \\
\hline None & $27.6(24)$ & $10.6(116)$ & Reference \\
\hline$<48 \mathrm{~h}$ & $35.6(31)$ & $27.6(303)$ & $0.5(0.3-0.9)$ \\
\hline$>48 \mathrm{~h}$ & $36.8(32)$ & $61.8(678)$ & $0.2(0.1-0.4)$ \\
\hline Use of $\mathrm{MgSO}_{4}$ & $18.4(16)$ & 25.3 (277) & $0.7(0.4-1.2)$ \\
\hline Multiple regnancy ${ }^{\mathrm{a}}$ & $14.9(13)$ & $28.6(314)$ & $0.4(0.2-0.8)$ \\
\hline Cesarean section & $54(47)$ & $57.9(635)$ & $1.2(0.8-1.8)$ \\
\hline \multicolumn{4}{|l|}{ Perinatal variables } \\
\hline Apgar $\leq 5$ at $5 \mathrm{~min}$ of age $\mathrm{a}^{\mathrm{a}}$ & $24.1(21)$ & $10.5(115)$ & $2.7(1.6-4.6)$ \\
\hline Cardiopulmonary resuscitation at birth ${ }^{\mathrm{a}}$ & $13.8(12)$ & $4.3(47)$ & $3.6(1.8-7.0)$ \\
\hline Admission hypothermia ${ }^{a}$ & $34.5(30)$ & $19.5(214)$ & $2.2(1.4-3.5)$ \\
\hline Out-born delivery & $11.5(10)$ & $6.6(72)$ & $1.8(0.9-3.7)$ \\
\hline \multicolumn{4}{|l|}{ Postnatal variables } \\
\hline \multicolumn{4}{|l|}{ Gestational age, weeks ${ }^{\mathrm{a}}$} \\
\hline $22-24$ & $9.2(8)$ & $6.3(69)$ & $2.4(1.1-5.3)$ \\
\hline $25-27$ & $50.6(44)$ & $28.4(312)$ & $2.9(1.8-4.6)$ \\
\hline $28-30$ & $40.2(35)$ & $65.3(716)$ & Reference \\
\hline Mean gestational age, weeks (SD) & $27.1(1.9)$ & $28.0(1.9)$ & \\
\hline Mean birth weight, grams (SD) & $1024(280)$ & $1142(321)$ & \\
\hline Males & $52(45)$ & $52(567)$ & \\
\hline Severe respiratory distress syndrome ${ }^{a}$ & $88.5(77)$ & $67(735)$ & $3.8(1.9-7.4)$ \\
\hline Cystic bronchopulmonary dysplasia ${ }^{a}$ & $28.7(25)$ & $15.8(173)$ & $2.2(1.3-3.5)$ \\
\hline \multicolumn{4}{|l|}{ Type of ventilation ${ }^{\mathrm{a}}$} \\
\hline None or non-invasive & $4.6(4)$ & $24.7(271)$ & Reference \\
\hline Positive pressure & $80.5(70)$ & $69.0(757)$ & $6.3(2.3-17.3)$ \\
\hline High frequency & $14.9(13)$ & $6.3(69)$ & $12.8(4.0-40.4)$ \\
\hline Hemodynamically significant PDA ${ }^{\mathrm{a}}$ & $40.2(35)$ & $23.5(258)$ & $2.2(1.4-3.4)$ \\
\hline Surgical ligation of PDA & $18.4(16)$ & $8.8(97)$ & $2.3(1.3-4.2)$ \\
\hline $\begin{array}{l}\text { Hypotension treated with inotropic } \\
\text { medication }^{\text {a }}\end{array}$ & $32.2(28)$ & $11.9(131)$ & $3.5(2.2-5.7)$ \\
\hline Culture positive sepsis ${ }^{\mathrm{a}}$ & $43.7(38)$ & $30.2(331)$ & $1.8(1.2-2.8)$ \\
\hline Systemic sepsis (culture negative) ${ }^{\mathrm{a}}$ & $55.2(48)$ & $39.7(436)$ & $1.9(1.2-2.9)$ \\
\hline CONS sepsis & $29.9(26)$ & $25.3(278)$ & $1.3(0.8-2.0)$ \\
\hline Non-CONS sepsis ${ }^{\mathrm{a}}$ & $13.8(12)$ & $4.8(83)$ & $3.2(1.6-6.2)$ \\
\hline Congenital pneumonia & $3.5(3)$ & $1.4(15)$ & $2.6(0.7-9.1)$ \\
\hline Fungal sepsis ${ }^{\mathrm{a}}$ & $5.8(5)$ & $0.6(7)$ & $9.5(3.0-30.6)$ \\
\hline Necrotizing enterocolitis ${ }^{a}$ & $6.9(6)$ & $2.5(27)$ & $2.9(1.2-7.3)$ \\
\hline
\end{tabular}


Table 1 (continued)

\begin{tabular}{llrr}
\hline Antenatal variables & WMI \% $(n=87)$ & $\begin{array}{l}\text { Non-WMI } \% \\
(n=1097)\end{array}$ & OR (95\% CI) \\
\hline Urinary tract infection $^{\mathrm{a}}$ & $14.9(13)$ & $6.5(71)$ & $1.2(0.6-2.5)$ \\
Prophylactic Indomethacin $_{\text {Intraventricular hemorrhage }}^{\mathrm{a}}$ & $10.3(9)$ & $8(88)$ & Reference \\
$\quad$ None & $35.5(31)$ & $75.1(824)$ & $1.5(0.7-2.9)$ \\
$\quad$ Grade I-II & $13.8(12)$ & $20.1(220)$ & $22.1(12.9-37.8)$ \\
$\quad$ Grade III-IV & $50.6(44)$ & $4.8(53)$ & \\
\hline
\end{tabular}

${ }^{a}$ Note: indicates $p<0.05, O R$ odds ratio, $C I$ confidence intervals

rupture of membranes were not different between the two groups.

Among the perinatal factors, the group with WMI was 3.6 times more likely to require cardiopulmonary resuscitation at birth compared with the non-WMI group. They were more likely to have apgar $\leq 5$ at $5 \mathrm{~min}$ of age (OR: 2.7). The admission hypothermia and out-born delivery were also more frequent in the WMI group. The newborns in WMI group were born at a lower mean gestation age and birth weight. However, the male to female distribution was similar in the two groups. Among the postnatal factors, the WMI group was more likely to have complications of severe respiratory distress syndrome (OR: 3.8), cystic bronchopulmonary dysplasia (OR: 2.2), grade III-IV intraventricular hemorrhage (OR: 22.1), hemodynamically significant patent ductus arteriosus (OR: 2.2), requirement for invasive positive pressure (OR: 6.3), and high frequency (OR: 12.8) ventilation, and requirement for inotropic medication (OR: 3.5). In addition, the WMI group demonstrated a high rate of culture positive sepsis (for organisms other than coagulase negative staphylococcal [CONS]) and fungal infections.

Table 2 summarizes the results of multivariate analysis for each of the three risk factor domains, as well as of the final model for WMI. In the adjusted analysis, histological chorioamnionitis, hypothermia on admission, invasive ventilatory support, hypotension treated with inotropic medication, culture positive bacterial sepsis (for organisms other than CONS) and fungal sepsis were all statistically significant risk factors for WMI. Antenatal steroids were associated with decreased risk. There was statistical significant increase in the use of antenatal steroids between epoch 1993-1999 and 2000-2006 $(p=0.02)$ but no significant difference was demonstrated between all three epochs for the use of antenatal steroids $(p=0.06)$.

Long-term outcome data were available in $94.4 \%$ of the patient population. Only two patients in cystic WMI group had no outcome information available. Mortality during first year of life was significantly higher in the cystic WMI group (Table 3). All neurodevelopmental morbidities except deafness were more prevalent in the WMI group. Motor disability was the most common disability in the cystic WMI compared to the non-WMI group, who had more cognitive delays. About half of the children with cystic WMI had none to mild disability.

\section{Discussion}

This retrospective population-based cohort study investigated the incidence, risk factors, and outcomes of cystic WMI in a very preterm cohort over two decades. The study identified a decreasing trend in the incidence of cPVL and porencephaly over time, when adjusted for gestational age and mortality. A number of clinical variables were found to be independently associated with cystic WMI. Cystic WMI was strongly predictive of cerebral palsy; however, half of the children with this injury had no significant disability. These findings have important implications for parental counselling and health care delivery. A low likelihood of selection bias owing to its population-based study design and a very low attrition rate are the main strengths of the study.

The incidence of cPVL ranges from 3 to 5\% in very lowbirth-weight infants based on ultrasound findings [10]' Literature on incidence of porencephaly is scarce. The current study showed a combined incidence of cPVL and porencepahly of $7.4 \%$ in VPIs based on ultrasonography. The unadjusted incidence of cystic WMI did not significantly change over three time epochs; this can be explained, in part, by the fact that the neonatal intensive care admission rates increased over time for babies born at $<28$ weeks' gestational age, the group most likely to experience WMI. An improvement in survival of VPIs may have also lead to increased detection of brain injury. However, after adjusting for gestational age and mortality, a significant decrease in the incidence of WMI was observed. These findings on decreasing incidence of over time have also been reported by others [18-20]. A better understanding of etio-pathogenesis of WMI and significant 
Table 2 Odds ratio and 95\% confidence intervals of independent variables associated with WMI: results of multivariate analysis

\begin{tabular}{|c|c|c|c|c|c|}
\hline Variables & $\begin{array}{l}\text { Model } 1 \text { (antenatal } \\
\text { variables only) }\end{array}$ & $\begin{array}{l}\text { Model } 2 \text { (perinatal } \\
\text { variables only) }\end{array}$ & $\begin{array}{l}\text { Model } 3 \text { (postnatal } \\
\text { variables only) }\end{array}$ & $\begin{array}{l}\text { Model } 4 \text { (antenatal } \\
+ \text { perinatal } \\
\text { variables) }\end{array}$ & $\begin{array}{l}\text { Model } 5 \text { (antenatal }+ \\
\text { perinatal + postnatal } \\
\text { variables) }\end{array}$ \\
\hline \multicolumn{6}{|l|}{ Antenatal } \\
\hline Chorioamnionitis & $2.2(1.2-4.1)$ & & & $2.2(1.1-4.2)$ & $2.2(1.1-4.2)$ \\
\hline \multicolumn{6}{|l|}{ Antenatal steroids } \\
\hline $24-48 \mathrm{~h}$ & $0.5(0.3-0.9)$ & & & $0.6(0.3-1.1)$ & $0.6(0.4-1.2)$ \\
\hline$>48 \mathrm{~h}$ & $0.2(0.1-0.4)$ & & & $0.3(0.2-0.5)$ & $0.3(0.2-0.6)$ \\
\hline \multicolumn{6}{|l|}{ Perinatal } \\
\hline Apgar $\leq 5$ at $5 \mathrm{~min}$ of age & & $2.6(1.5-4.5)$ & & $2.2(1.3-3.8)$ & $1.5(0.8-2.6)$ \\
\hline Hypothermia at admission & & $2.0(1.2-3.2)$ & & $1.8(1.1-3.0)$ & $1.7(1.1-2.7)$ \\
\hline \multicolumn{6}{|l|}{ Postnatal } \\
\hline \multicolumn{6}{|l|}{ Type of ventilation } \\
\hline None or non-invasive & & & Reference & & Reference \\
\hline Positive pressure & & & $5.2(1.9-14.6)$ & & $4.1(1.5-11.6)$ \\
\hline High frequency & & & $6.2(1.8-21.0)$ & & $4.9(1.4-17.1)$ \\
\hline Non-CONS sepsis & & & $2.1(1.0-4.3)$ & & $2.3(1.1-4.6)$ \\
\hline Fungal sepsis & & & $4.4(1.3-14.8)$ & & $4.4(1.3-15.5)$ \\
\hline $\begin{array}{l}\text { Hypotension treated with } \\
\text { inotropes }\end{array}$ & & & $2.3(1.3-4.1)$ & & $1.9(1.1-3.4)$ \\
\hline
\end{tabular}

Note: models 1, 2, and 3 were derived from antenatal, perinatal, and postnatal variables (mentioned in Table 1) respectively. Variables with a $p$ value of $<0.20$ in univariate analysis were entered into these models and retained in the model if $p$ value was $<0.05$. Model 4 was generated by adding the block of variables derived from the perinatal model to the antenatal model. Variables derived from postnatal model were added to model 4 resulting in the final model 5. The final model has variables of antenatal, perinatal, and postnatal origin

advances in perinatal and neonatal care of preterm population including an increase in the use of antenatal steroids as documented in the current study has likely contributed to this change.

The pathogenesis of PVL is complex and multiple mechanisms of vascular, circulatory, or inflammatory in origin have been held responsible in literature [4]. Chorioamnionitis has emerged as one of the important risk factors for WMI in preterm infants with several studies and three meta-analysis demonstrating such an association $[10,21-24]$. The underlying hypothesis is that the infection triggers a fetal inflammatory response with release of proinflammatory cytokines. This results in onset of an inflammatory cascade, which exerts a harmful effect on the preterm developing brain through following mechanisms: (1) direct toxic effect on oligodendrocytes and their progenitors in the white matter; (2) initiating a microglial response with free radical generation and glutamatemediated excitoxicity, and (3) increasing permeability of the blood-brain barrier and impairment of cerebral blood flow. However, in a recent literature review by Chau et al. [25], authors stated that the relationship between chorioamninoitis and brain injury is not yet certain and any definitive conclusions regarding causation cannot be drawn owing to the presence of heterogeneity and methodological limitations across published studies. The review also reported that one of the major limitations of published studies was the criteria used to define chorioamnionitis. In the majority of studies, chorioamnionitis was diagnosed based on the clinical judgment. Histological criteria, which has been described as the gold standard for diagnosis of chorioamnionitis was infrequently used. In the current study, histological chorioamnionitis was independently associated with an increased risk for WMI; although a causal relationship cannot be inferred owing to the retrospective nature of the study.

Both premature and prolonged rupture of membranes increase the likelihood of intrauterine infection and have also been identified as risk factors for cPVL in literature [9, 10, 21]. However, this relationship was not observed in the current study. This difference in study findings may be related to the population-based cohort study design with a low risk of selection bias compared to most case-control or non population-based studies published in literature.

This study showed that both postnatal infections and chorioamnionitis were independently associated with cystic WMI. In a prospective study on non-cystic WMI in preterm infants born between 24-32 weeks of gestation, authors observed that postnatal infection was a more important risk factor than histological chorioamnioitis [26]. In contrast, the 
Table 3 Death and neurodevelopmental outcomes at 18-36 months of age in WMI vs non-WMI group

\begin{tabular}{|c|c|c|c|}
\hline Outcome & WMI \% (n/total) & Non-WMI \% (n/total) & OR $(95 \% \mathrm{CI})$ \\
\hline $\begin{array}{l}\text { Death } 42- \\
364 \text { days }\end{array}$ & $5.8(5 / 85)$ & $1.5(15 / 1030)$ & $4.2(1.2-12.6)$ \\
\hline Normal outcome $\mathrm{a}^{\mathrm{a}}$ & $15(12 / 80)$ & $78.2(791 / 1011)$ & $0.05(0.02-0.09)$ \\
\hline Overall disability $^{\mathrm{a}}$ & $85(68 / 80)$ & $21.8(220 / 1011)$ & $20.4(10.7-41.2)$ \\
\hline Mild disability ${ }^{\mathrm{a}}$ & $31.3(25 / 80)$ & $13.8(140 / 1011)$ & $2.8(1.6-4.8)$ \\
\hline $\begin{array}{l}\text { Moderate } \\
\text { disability }^{\text {a }}\end{array}$ & $21.3(17 / 80)$ & $4.5(45 / 1011)$ & $5.8(2.9-11.0)$ \\
\hline Severe disability ${ }^{\mathrm{a}}$ & $32.5(26 / 80)$ & $3.5(35 / 1011)$ & $13.4(7.2-24.7)$ \\
\hline $\begin{array}{l}\text { Cerebral palsy (all } \\
\text { level) }{ }^{a}\end{array}$ & $81.3(65 / 80)$ & $6.2(63 / 1011)$ & $65.2(34.1-129)$ \\
\hline $\begin{array}{l}\text { Cerebral palsy } \\
\text { level } 1^{\text {a }}\end{array}$ & $36.7(29 / 79)$ & $4.7(48 / 1011)$ & $11.6(6.5-20.6)$ \\
\hline $\begin{array}{l}\text { Cerebral palsy } \\
\text { level } 2-3^{\mathrm{a}}\end{array}$ & $24(19 / 79)$ & $1.1(11 / 1011)$ & $28.8(12.3-69.6)$ \\
\hline $\begin{array}{l}\text { Cerebral palsy } \\
\text { level } 4-5\end{array}$ & $19(15 / 79)$ & $(<5 / 1011)$ & NA \\
\hline Blindness & $9(7 / 78)$ & $(<5 / 1030)$ & NA \\
\hline Deafness & $(0 / 78)$ & $0.9(9 / 1030)$ & NA \\
\hline Mean MDI score ${ }^{a}$ & $78.78 \pm 28.10(76)$ & $96.47 \pm 18.22(1030)$ & $-17.7(-22.1$ to -13.2$)$ \\
\hline $\mathrm{MDI}<85^{\mathrm{a}}$ & $51.3(39 / 76)$ & $21.3(219 / 1030)$ & $3.9(2.4-6.4)$ \\
\hline $\mathrm{MDI}<70^{\mathrm{a}}$ & $36.8(28 / 76)$ & $7.4(76 / 1030)$ & $7.3(4.2-12.7)$ \\
\hline $\mathrm{MDI}<55^{\mathrm{a}}$ & $25(19 / 76)$ & $2.9(30 / 1030)$ & $11.1(5.5-21.7)$ \\
\hline
\end{tabular}

${ }^{a}$ Note: indicates $p<0.05, O R$ odds ratio, $C I$ confidence intervals, $N A$ not assessed current study emphasizes the importance of both in utero and postnatal infections in the pathogenesis of cystic WMI, and therefore, underscores the need of effective strategies for prevention, early diagnosis and management of both conditions. Further, it was observed that odds of cystic WMI significantly increased with non-CONS and fungal type of infections. Non-cystic WMI is clearly more frequently reported in preterm newborns with postnatal bacterial and fungal infections [27-29]. However, to the best of our knowledge, an association between cystic WMI and postnatal bacterial and fungal infections has not been demonstrated before. This association was observed independently of the hypotension suggesting the fact that a host of inflammatory mediators are likely responsible for this insult.

An association between multiple births and PVL has been described in some studies, whereas others have failed to establish this notion [30]. The factors predisposing to WMI in multiple gestation include intrauterine growth retardation, imbalanced placental blood supply, twin-totwin transfusion, and intrauterine death of the co-twin. This association was not observed in the current study. In contrast, multiple gestation was associated with lower odds of having WMI on univariate analysis, but this association failed to achieve statistical significance on multivariate analysis. The protective effect of antenatal corticosteroids and association of hypotension and mechanical ventilation with cystic WMI is documented in the literature and this was also supported by study findings [12, 31, 32].

Hypothermia on admission was identified as an independent risk factor for WMI. This is consistent with the publication from the Canadian Neonatal Network reporting an association between severe neurological injury and admission temperature of $<36.5 \mathrm{C}$ [33]. Authors speculate that the WMI in this setting might be mediated through IVH, which can be a consequence of hypothermia. IVH has also been linked to cPVL in literature. In a retrospective cohort study on preterm babies born before 30 weeks of gestation, authors reported that the presence of IVH grade 3 and 4 significantly increased the risk of cPVL, even after adjusting for potential confounders [34]. The current study also observed a very strong association between IVH and cystic WMI, partly owing to the fact that IVH is a predisposing factor for cystic porencephaly. However, our observations also raise the possibility of IVH being a causal link to cPVL.

This study also reported population-based long-term outcomes of cystic WMI in VPI. Cerebral palsy was documented in $81 \%$ of the cystic WMI group, which is consistent with the published reports [6]. However, half of the children with cystic brain injury did not have significant long-term disability, an important observation that 
emphasizes the limited utility of neuroimaging in predicting the functional outcomes. It is also possible that the neuroplasticity of the premature brain may also play a role. As cystic WMI is usually diagnosed late during a baby's course in the NICU and often, these babies are relatively medically stable, the results of this study have important implications for counselling the families in the NICU. A very pessimistic approach may not be very helpful in the cases, where withdrawal of life support is not an option. Of note, cPVL or porencephaly did not increase the risk of deafness in survivors in the current study. This is in contrast to findings by Cooke, who reported deafness in 6 out of $66(9 \%)$ preterm infants with cystic brain lesions [35]. The difference in study findings may be related to different population characteristics or different patterns of injury.

In addition to a population-based study design, other strengths of the study include comprehensive information on numerous clinical variables of interest over two decades, which allowed the evaluation of incidence, relevant risk factors, and neurodevelopmental outcomes. In addition, although magnetic resonance imaging is considered superior for diagnosing subtle WMI, the current study looked at more obvious cystic WMI, which can be reliably diagnosed by serial cranial ultrasounds [6]. Also, a very high followup rate in the current study enabled an accurate reflection of the outcomes. The limitation of the study lies in its inability to distinguish cPVL from porencephaly owing to its database design. However, it still provides clinically relevant and useful information, as both these conditions are closely associated with adverse neurodevelopmental outcomes. Retrospective studies are limited to available data, and there may be other variables relevant to the present study that are not routinely captured in the PFUP database. Especially, the study is limited in its ability to provide information about severity and grading of the cystic injury. Furthermore, as the study was conducted over two decades, it is possible that the clinical care and hence the risk factors may have changed over this time period; however, the time epochs were not identified as a significant risk factor for WMI.

In conclusion, this population-based cohort study reported a decreasing trend and identified a number of clinically relevant factors associated with $\mathrm{CPVL}$ or porencephaly in VPI. These findings have important implications in the delivery of perinatal and neonatal care to reduce the incidence of WMI and consequent, neurological morbidity. The study results are also useful for more informative parent counseling in a population-based setting

\section{Compliance with ethical standards}

Conflict of interest The authors declare that they have no conflict of interest.

\section{References}

1. Marlow N, Wolke D, Bracewell MA, Samara M. Neurologic and developmental disability at six years of age after extremely preterm birth. N Engl J Med. 2005;352:9-19.

2. Synnes A, Luu TM, Moddemann D, Church P, Lee D. Canadian Neonatal Network and the Canadian Neonatal Follow-Up Network. Determinants of developmental outcomes in a very preterm Canadian cohort. Arch Dis Child Fetal Neonatal Ed. 2017;102: F235-F234.

3. Back SA. White matter injury in the preterm infant: pathology and mechanisms. Acta Neuropathol. 2017;134:331-49.

4. Bass WT. Periventricular leukomalacia. Neoreviews. 2011;12:76-84.

5. Deng W, Pleasure J, Pleasure D. Progress in periventricular leukomalacia. Arch Neurol. 2008;65:1291-5.

6. De Vries LS, Van Haastert IL, Rademaker KJ, Koopman C, Groenendaal F. Ultrasound abnormalities preceding cerebral palsy in high-risk preterm infants. J Pediatr. 2004;144:815-20.

7. Al Tawil KI, El Mahdy HS, Al Rifai MT, Tamim HM, Ahmed IA, Al Saif SA. Risk factors for isolated periventricular leukomalacia. Pediatr Neurol. 2012;46:149-53.

8. Resch B, Neubauer K, Hofer N, Resch E, Maurer U, Haas J, et al. Episodes of hypocarbia and early-onset sepsis are risk factors for cystic periventricular leukomalacia in the preterm infant. Early Hum Dev. 2012;88:27-31.

9. Bauer M, Fast C, Haas J, Resch B, Lang U, Pertl B. Cystic periventricular leukomalacia in preterm infants: an analysis of obstetric risk factors. Early Hum Dev. 2009;85:163-9.

10. Perlman JM, Risser R, Broyles RS. Bilateral cystic periventricular leukomalacia in the premature infant: associated risk factors. Pediatr. 1996;97:822-7.

11. Liu J, Li J, Qin GL, Chen YH, Wang Q. Periventricular leukomalacia in premature infants in mainland China. Am J Perinatol. 2008;25:535-40.

12. Shankaran S, Langer JC, Kazzi SN, Laptook AR, Walsh M. National Institute of Child Health and Human Development Neonatal Research Network. Cumulative index of exposure to hypocarbia and hyperoxia as risk factors for periventricular leukomalacia in low birth weight infants. Pediatr. 2006;118:1654-9.

13. Murase M, Ishida A. Early hypocarbia of preterm infants: its relationship to periventricular leukomalacia and cerebral palsy, and its perinatal risk factors. Acta Paediatr. 2005;94:85-91.

14. Bax M, Goldstein M, Rosenbaum P, Leviton A, Paneth N, Dan B, et al. Proposed definition and classification of cerebral palsy, April 2005. Dev Med Child Neurol. 2005;47:571-6.

15. Palisano R, Rosenbaum P, Walter S, Russell D, Wood E, Galuppi B. Development and reliability of a system to classify gross motor function in children with cerebral palsy. Dev Med Child Neurol. 1997;39:214-23.

16. Moore T, Johnson S, Haider S, Hennessy E, Marlow N. Relationship between test scores using the second and third editions of the Bayley Scales in extremely preterm children. J Pediatr. 2012;160:553-8.

17. Hollingshead AB. Four-factor index of social status [Doctoral thesis]. New Haven, CT: Yale University; 1975.

18. Groenendaal F, Termote JU, van der Heide-Jalving M, van Haastert IC, de Vries LS. Complications affecting preterm neonates from 1991 to 2006: what have we gained? Acta Paediatr. 2010;99:354-8.

19. Hamrick SE, Miller SP, Leonard C, Glidden DV, Goldstein R, Ramaswamy V, et al. Trends in severe brain injury and neurodevelopmental outcome in premature newborn infants: the role of cystic periventricular leukomalacia. J Pediatr. 2004;145:593-9.

20. van Haastert IC, Groenendaal F, Uiterwaal CS, Termote JU, van der Heide-Jalving M, Eijsermans MJ, et al. Decreasing incidence 
and severity of cerebral palsy in prematurely born children. J Pediatr. 2011;159:86-91. e1

21. Resch B, Vollaard E, Maurer U, Haas J, Rosegger H, Müller W. Risk factors and determinants of neurodevelopmental outcome in cystic periventricular leucomalacia. Eur J Pediatr. 2000;159:663-70.

22. Wu YW. Systematic review of chorioamnionitis and cerebral palsy. Ment Retard Dev Disabil Res Rev. 2002;8:25-29.

23. Wu YW, Colford JM. Chorioamnionitis as a risk factor for cerebral palsy: a meta-analysis. JAMA. 2000;284:1417-24.

24. Shatrov JG, Birch SC, Lam LT, Quinlivan JA, McIntyre S, Mendz GL. Chorioamnionitis and cerebral palsy: a meta-analysis. Obstet Gynecol. 2010;116:387-92.

25. Chau V, McFadden DE, Poskitt KJ, Miller SP. Chorioamnionitis in the pathogenesis of brain injury in preterm infants. Clin Perinatol. 2014;41:83-103.

26. Chau V, Poskitt KJ, McFadden DE, Bowen-Roberts T, Synnes A, Brant R, et al. Effect of chorioamnionitis on brain development and injury in premature newborns. Ann Neurol. 2009;66:155-64.

27. Inomata K, Mizobuchi M, Tanaka S, Iwatani S, Sakai H, Yoshimoto $\mathrm{S}$, et al. Patterns of increases in interleukin-6 and C-reactive protein as predictors for white matter injury in preterm infants. Pediatr Int. 2014;56:851-5.

28. Barton M, O’Brien K, Robinson JL, Davies DH, Simpson K, Asztalos E, et al. Invasive candidiasis in low birth weight preterm infants: risk factors, clinical course and outcome in a prospective multicenter study of cases and their matched controls. BMC Infect Dis. $2014 ; 14: 327$.
29. Adams-Chapman I, Bann CM, Das A, Goldberg RN, Stoll BJ, Walsh MC. et al.Eunice Kennedy Shriver National Institutes of Child Health and Human Development Neonatal Research Network Neurodevelopmental outcome of extremely low birth weight infants with Candida infection. J Pediatr. 2013;163:961.

30. Resch B, Resch E, Freidl T, Maurer U, Haas J, Müller W. Preterm twin and triplet pregnancies are at increased risk for the development of cystic periventricular leukomalacia. Eur J Paediatr Neurol. 2013;17:148-52.

31. Baud O, Foix-L'Helias L, Kaminski M, Audibert F, Jarreau PH, Papiernik E, et al. Antenatal glucocorticoid treatment and cystic periventricular leukomalacia in very premature infants. $\mathrm{N}$ Engl J Med. 1999;341:1190-6.

32. Riddle A, Maire J, Cai V, Nguyen T, Gong X, Hansen K, et al. Hemodynamic and metabolic correlates of perinatal white matter injury severity. PLoS ONE. 2013;8:e82940.

33. Lyu Y, Shah PS, Ye XY, Warre R, Piedboeuf B, Deshpandey A, et al. Association between admission temperature and mortality and major morbidity in preterm infants born at fewer than 33 weeks' gestation. JAMA Pediatr. 2015;169:e150277.

34. Kusters CD, Chen ML, Follett PL, Dammann O. "Intraventricular" hemorrhage and cystic periventricular leukomalacia in preterm infants: how are they related? J Child Neurol. 2009;24:1158-70.

35. Cooke RW. Early and late cranial ultrasonographic appearances and outcome in very low birthweight infants. Arch Dis Child. 1987;62:931-7. 